\title{
Performance Comparison of Daubechies, Biorthogonal and Haar Transform for Grayscale Image Compression
}

\author{
VaruneshKumar Mishra \\ M.Tech. Scholar \\ ECE DepartmentSHIATS-DU \\ Allahabad, India
}

\author{
Anil Kumar \\ Assistant Professor ECE \\ DepartmentSHIATS-DU \\ Allahabad, India
}

\author{
A.K. Jaiswal \\ Professor,H.O.D.,ECE \\ Department \\ SHIATS-DU Allahabad, India
}

\begin{abstract}
Image compression is process to remove the redundant information from the image so that only essential information are stored the storage size, transmission bandwidth and transmission time. In this paper comparative analysis of gray scale image compression is done by three different methods that are Daubechies Transform, Haar Transform and Biorthogonal Transform. Experimental results demonstrate that the Daubechies algorithm is more efficient for PSNR and MSE while Haar Wavelet Transform provides the better compression ratio.
\end{abstract}

\section{Keywords}

Image compression, Haar Transform, Biorthogonal

Transform, Daubechies Transform, PSNR, MSE, CR.

\section{INTRODUCTION}

Image compression is important for many applications that involve large data storage, transmission and retrieval. The compressed image is represented by less number of bits as compared to original. The required storage size is also reduced, consequently maximum images be stored and it is transferred in faster way to save the time and transmission bandwidth. There are many applications where the image compression are used to effectively increased efficiency and performance. Applications are like health industries, retail stores, security industries, museums and galleries etc. To analysis the parameters of image compression PSNR and $\mathrm{CR}$ is important parameters. It gives synthetic performance of the compression of images. Image is a 2 dimensional signal represented by digital system. A digital image is basically 2dimensional array of pixels. Basically compressing an image is different from compressing digital data.

\subsection{Mean Square Error (MSE)}

It is a risk function, an average of the square of the difference between the desired response and the actual system output.

MSE $=\frac{1}{M N} \sum_{Y=1}^{M} \sum_{X=1}^{N}\left[I(X, Y)-I^{\prime}\left(X^{\prime}, Y^{\prime}\right)\right]^{2}$

Where $m x n$ is the size of the image and $I(X, Y)$ is the original input image and I' ( $\mathrm{X}^{\prime}, \mathrm{Y}^{\prime}$ ) is the compressed image.

\subsection{Peak Signal To Noise Ratio}

PSNR defined the ratio of maximum possible power and the power of corrupting noise in an image. The PSNR is defined as-
$\mathrm{PSNR}=20 * \log 10(255 /$ sqrt $(\mathrm{MSE})$

\section{VARIOUS STEPS IN IMAGE COMPRESSION}

Fig. 1 describes the fundamental steps of an image Compression system. Encoder creates some symbols from the input data to represent the image. Image $f^{n}(x, y)$ represent an approximation of the input data that results from compressing and subsequently decompressing the input image.

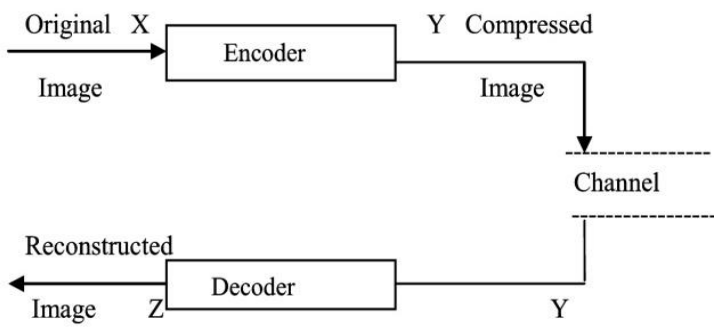

Fig.1 Different steps in an image compression system.

The formula of compression ratio is following:

$\mathrm{CR}=\mathrm{n} 1 / \mathrm{n} 2$

Where $\mathrm{n} 1$ represent the bits of information carrying in the original image and $\mathrm{n} 2$ denote the bits of information carrying in the compressed image respectively. A compression ratio of 10:1 denotes that the original image has 10 information bits for every 1 bit in the compressed image.

\section{PRINCIPLE AND PROCESS OF DT (DAUBECHIES TRANSFORM)}

The Daubechies wavelets, based on the work of Ingrid Daubechies, are a family of orthogonal wavelets defining a discrete wavelet transform and characterized by a maximal number of vanishing moments for some given support. The Daubechies transforms calculate the running averages and differences via scalar products with scaling signals and wavelets the only difference between them consists in how these scaling signals and wavelets are defined. Daubechies transforms has balanced frequency responses but non-linear phase responses. Daubechies transform use overlapping windows, so the high frequency coefficient spectrum reflects all high frequency changes. Therefore Daubechies transform is useful in compression and noise removal of audio signal processing.

To define Daubechies-based wavelets, The scaling function is the solution of the dilation equation, 


$$
\phi(x)=\sqrt{2} \sum_{k=0}^{L-1} h_{k} \phi(2 x-k)
$$

Where $\phi(\mathrm{x})$ is normalized $\int \phi(\mathrm{x}) \mathrm{dx}=1$, and the wavelet $\Psi(\mathrm{x})$ is defined in terms of the scaling function,

$$
\psi(x)=\sqrt{2} \sum_{k=0}^{L-1} g_{k} \phi(2 x-k)
$$

Both the scaling sequence (Low Pass Filter) and the wavelet sequence (Band Pass Filter) will here be normalized to have summed equal 2 and sum of squares equal 2.

\section{PRINCIPLE OF HT (HAAR TRANSFORM)}

Compression through Haar transform is an efficient way to perform both lossless and lossy image compression. It relies on averaging and differencing values in an image matrix to produce a matrix which is sparse or nearly sparse. Haar Transform decomposes each signal into two components, one is called average (approximation) or trend and the other is known as difference or fluctuation. The first level of approximation $\mathrm{a}_{\mathrm{m}}$ is defined as

$$
a_{m}=\frac{X_{2 m-1}+X_{2 m}}{\sqrt{2}}
$$

For $m=1,2,3 \ldots \ldots \mathrm{N} / 2$ where $\mathrm{x}$ is the input signal. The multiplication of $\sqrt{2}$ indicates that the HT preserves the energy of the signal. The values of $\alpha^{1}$ represent the average of successive pairs of $x$ value. The first level detail $d^{1}=\left(d_{1}, d_{2}\right.$ ,.... $\left.\mathrm{dN}_{/ 2}\right)$ is defined as

$$
d_{m}=\frac{X_{2 m-1}-X_{2 m}}{\sqrt{2}}
$$

For $m=1,2,3 \ldots \ldots . N / 2$ the values of $d^{1}$ represents the difference of successive pairs of $x$ value.

The Haar function is defined as

$h_{k}(\mathrm{t})=\frac{1}{\sqrt{N}}\left\{\begin{array}{cl}2^{p / 2} & (q-1) / 2^{p} \leq t<(q-0.5) / 2^{p} \\ -2^{p / 2} & (q-0.5) / 2^{p} \leq t<q / 2^{p} \\ 0 & \text { otherwise }\end{array}\right.$

From the above equation, one can see that $p$ determines the amplitude and width of the non-zero part of the function, while $q$ determines the position of the non-zero part of the Haar function

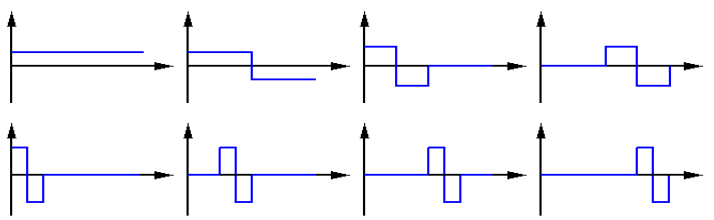

Fig.2 Haar functions for composing 8-point Haar transform matrix

Haar Transform decomposes image in $8 \times 8$ matrixes. The process can be generalized to $2^{\mathrm{K}} \times 2^{\mathrm{K}}$ matrix, where is $\mathrm{k}$ integer positive number. One divides corresponding matrix into $8 \times 8$ blocks and considers blocks like separate matrices. Process should be repeat on each block matrix. If there are $2^{\mathrm{K}}$ elements in row in matrix, then the transformation process row of matrix will consist of $\mathrm{k}$ steps.

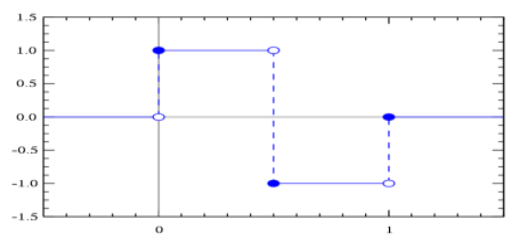

Fig.3 The Haar wavelet

\section{PRINCIPLEOF BIORTHOGONAL TRANSFORM (BT)}

Orthogonality condition allow to gain greater flexibility in the construction of wavelet bases. Biorthogonal wavelets are families of compactly supported symmetric wavelets. In the biorthogonal case, there are two scaling functions that may generate different multiresolution analysis, and accordingly two different wavelet functions.

The dual scaling and wavelet functions have the following properties:

1. They are zero outside of a segment.

2. The calculation algorithms are maintained, and thus very simple.

3. The associated filters are symmetrical.

4. The functions used in the calculations are easier to build numerically.

The approximation spaces $\mathrm{Vj}-1 \subset \mathrm{Vj} \subset \mathrm{Vj}+1$ and an orthogonal decomposition $\mathrm{Vj}+1=\mathrm{Vj} \oplus \mathrm{Wj}$ leads to a reconstruction and decomposition scheme relying on two filters, say sequences $h_{n}$ and $g_{n}$. Let $f_{k}, g_{k} \in H$. so that the sequences $\mathrm{f}_{\mathrm{k}}$ and $\mathrm{g}_{\mathrm{k}}$ are biorthogonal if $\left(\mathrm{h}_{\mathrm{k}}, \mathrm{g}_{\mathrm{k}}\right)=\delta_{\mathrm{j} \mathrm{k}}$.

\section{METHODOLOGY}

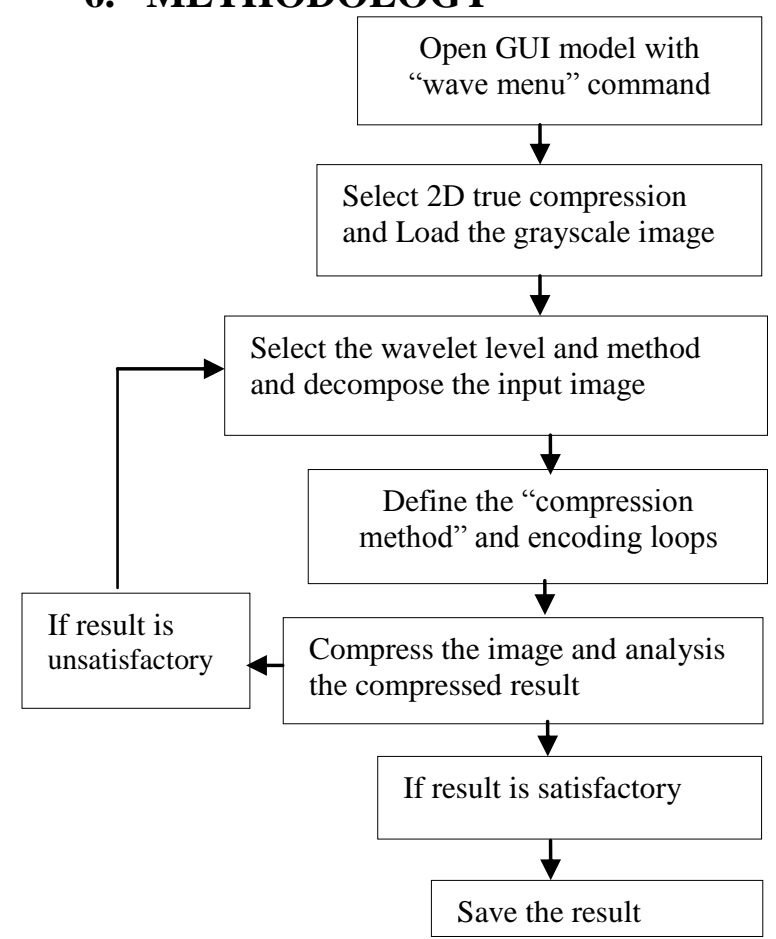

Flow chart of compression using DT, HT and BT 


\section{RESULT \& DISCUSSION}

Grayscale image compression using BT, HT and DT can be done through MATLAB. The following image compression results perform the comparison between Daubechies, Biorthogonal and Haar Transform. Each method has its own specification. Here is the table which shows the difference between each method. Following experimental result show that Daubechies method gives the better PSNR (39.68) and MSE value (7.001) value while Haar method provides the much CR value $(33.48 \%)$.

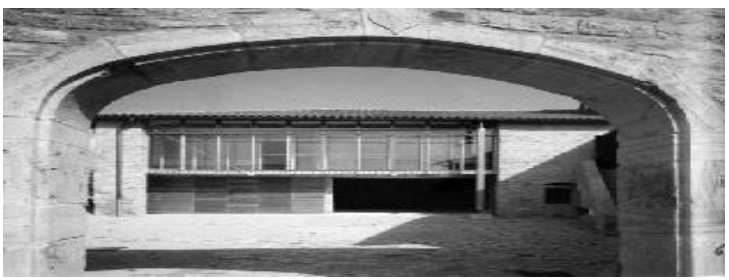

Fig.4. Original image

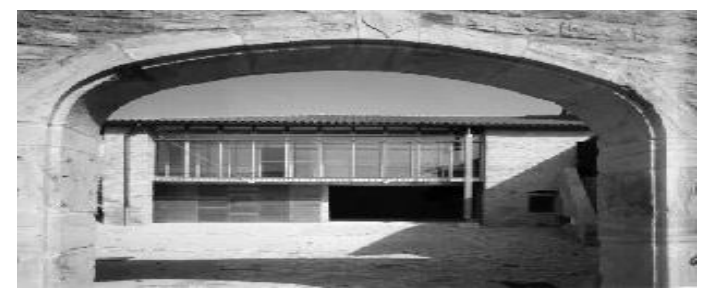

Fig.5. Compressed image by Daubechies method

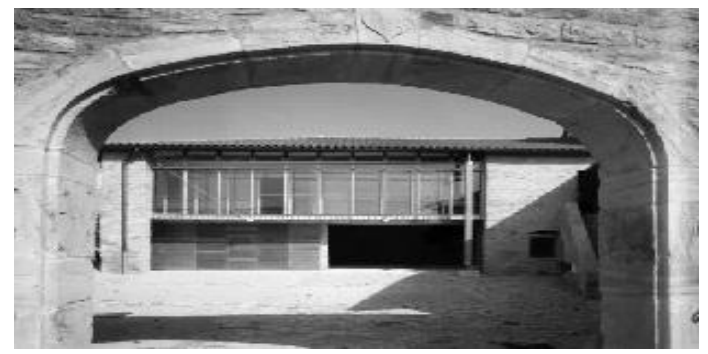

Fig.6. Compressed image by Haar method

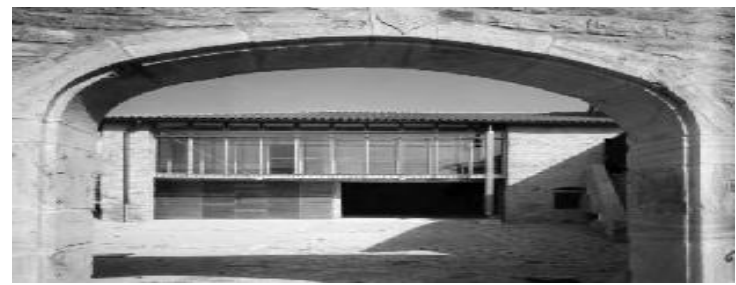

Fig.7. Compressed image by Biorthogonal method

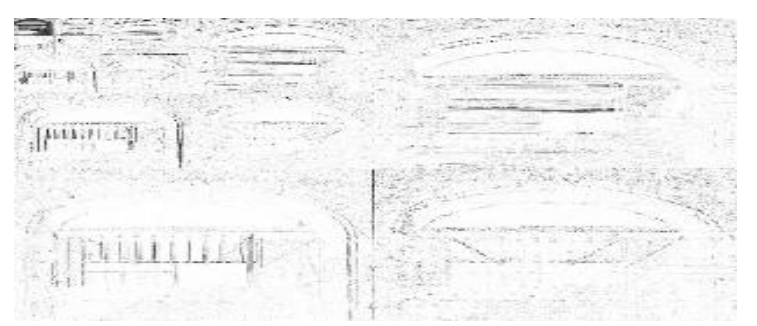

Fig.8. Image Decomposition of Original Image

Table For Result Comparison

\begin{tabular}{|l|l|l|l|}
\hline & DT & HT & BT \\
\hline MSE & 7.001 & 7.523 & 7.052 \\
\hline PSNR & 39.68 & 38.74 & 39.43 \\
\hline CR & $27.09 \%$ & $33.48 \%$ & $22.63 \%$ \\
\hline
\end{tabular}

\section{CONCLUSION}

This paper compares Daubechies Transform, Haar Transform and Biorthogonal Transform schemes for same grayscale image through matlab. Simulation results indicate that image compression based on Daubechies transform could retain more information of image than Biorthogonal transform and Haar Transform.

\section{REFERENCES}

[1] P. Ravi raj, 2007 "The Modified 2D-Haar Wavelet Transformation in Image Compression".

[2] S.S. Tamboli, 2013, "Image Compression Using Haar Wavelet Transform ".

[3] Hermina Alajbegović, 2009 "Image Compression Using the Haar Wavelet Transform".

[4] Colm Mulcahy, 2007 " Image compression using the Haar wavelet transform".

[5] Kamrul Hasan Talukder, 2007 "Haar Wavelet Based Approach for Image Compression and Quality Assessment of Compressed Image".

[6] Telagarapu P., Jagan N. V. 20011 "Image Compression Using DCT and Wavelet Transformations".

[7] Rajesh K. Yadav, S.P. Gangwar, 2012 "Study and analysis of wavelet based image compression techniques".

[8] Sonja Grgic, 2001 "Performance Analysis of Image Compression Using Wavelets".

[9] A. Ghorpade,2011“Image Compression Using Haar

[10] transformandModified Fast Haar Wavelet Transfo".Rado mir S. Stankovi. 2003 "The Haar wavelet transform: its status and achievements".

[11] Anuj Bhardwaj and Rashid Ali, 2009 "Image Compression Using Modified Fast Haar Wavelet Transform".

[12] Zunera Idrees, 2006 "Image Compression by Using Haar Wavelet Transform and Singular Value Decomposition".

[13] Mohammed A. Salem, 2009 "Daubechies Versus Biorthogonal Wavelets for Moving Object Detection in Traffic Monitoring Systems". 